\title{
Dietary Supplements in Management and Prevention of Chronic Disease
}

\section{Michael B Zemel*}

The University of Tennessee, Knoxville, Tennessee, USA

Dietary supplement use for both general health and specific applications has been steadily growing, and more than $50 \%$ of adults in the US now use at least one supplement daily. This is accompanied by an often well-deserved dose of healthy skepticism on the part of the health care and research communities. However, despite our ambivalence, the pages of many biomedical journals, including this one, continue to fill with reports demonstrating robust in vitro and in vivo bioactivity of select food components and complex extracts as well as a smaller number of clinical trials validating disease-specific applications.

So, with an abundance of high quality scientific literature supporting significant bioactivity of multiple food components, what are the reasons for a generalized distrust of dietary supplements for prevention and management of disease? Clearly one component must be a discomfort with the overwhelming abundance of minimally regulated, poorly supported (or completely unsupported) claims and implications driven by marketers rather than by sound science. However, even conservative, evidence based claims are generally met with skepticism in the nutritional science community. This is a community with a cornerstone belief in the value of healthy diets (despite our disagreements surrounding what constitutes a healthy diet), rather than utilizing food components in a medicinal fashion.

And yet, we continue to investigate food-derived bioactivity and to proclaim its potential value in our grant applications and our published manuscripts. It is now time to take an active role in translating these studies, both positive and negative, into useful, practical approaches that can be utilized as a benchmark for assessing supplement claims. Let us use Resveratrol as an example. This polyphenol, found in the skin of red grapes and other fruits, has been widely demonstrated to serve as a Sirt1 activator, mimicking the effects of energy restriction on lifespan and multiple metabolic outcomes, including diabetes [1-5]. However, most of these effects are only achieved at very high doses that are difficult to obtain in humans, while limited bioavailability and rapid metabolism make achieving therapeutically effective plasma levels a significant challenge. Consequently, promising results from cellular and rodent studies with very high concentrations of this polyphenol are not easily translated to human outcomes, and more reasonable doses have been reported to have little or no effect [6]. However, this has not prevented extensive marketing of resveratrol at doses unlikely to provide meaningful effects. Notably, some (albeit a minority) dietary supplements offer demonstrated efficacy with the doses used in the formulations, but these risks are getting lost in the larger universe of questionable supplements.

The Nutritional Science community has a role to play in helping both health professionals and consumers that navigate this universe. As a starting point, here are some modest principles to be considered for benchmarking supplements:

- Claims must be based upon sound science, using accepted, standardized approaches;

- Clear scientific rationale and cellular mechanism of action must be demonstrated;

- In vitro data is necessary, but by no means sufficient. The literature has numerous examples of promising approaches demonstrated in the in vitro systems that fail when applied to animals and humans.
- In vivo demonstration of safety and efficacy in the target population must be presented using the same doses as used in the products when marketed. The nutrition literature has demonstrated the efficacy of several plant derivatives in the prevention/management of chronic diseases using doses that are difficult or impossible to obtain; those same derivatives are then marketed at much lower doses with reference made to the "high dose" literature. Resveratrol supplements are one such example of this problem, and cinnamon applications in diabetes are another. These create both practitioner and consumer confusion, as there is indeed validation studies, but not using the doses in the supplements.

- Key data must be available for scrutiny in peer-reviewed scientific publications for public and scientific scrutiny.

Consistent application of these simple benchmark principles by the Nutritional Science community is the first step towards restoring the confidence of both clinicians and consumers that there is appropriate alignment between the data and the claims. Translating our science into practical use demands that this scientific community undertake the task of utilizing this framework to systematically evaluate dietary supplements and periodically provide the results of this evaluation to the broader community.

\section{References}

1. Baur JA, Pearson KJ, Price NL, Jamieson HA, Lerin C, et al. (2006) Resveratrol improves health and survival of mice on a high-calorie diet. Nature 444: 337 342

2. Lagouge M, Argmann C, Gerhart-Hines Z, Meziane H, Lerin C, et al. (2006) Resveratrol improves mitochondrial function and protects against metabolic disease by activating SIRT1 and PGC-1alpha. Cell 127: 1109-1122.

3. Park SJ, Ahmad F, Philp A, Baar K, Williams T, et al. (2012) Resveratrol ameliorates aging-related metabolic phenotypes by inhibiting cAMP phosphodiesterases. Cell 148: 421-433.

4. Price NL, Gomes AP, Ling AJ, Duarte FV, Martin-Montalvo A, et al. (2012) SIRT1 is required for AMPK activation and the beneficial effects of resveratrol on mitochondrial function. Cell Metab 15: 675-690.

5. Baur JA, Sinclair DA (2006) Therapeutic potential of resveratrol: the in vivo evidence. Nat Rev Drug Discov 5: 493-506.

6. Yoshino J, Conte C, Fontana L, Mittendorfer B, Imai SI, et al. (2012) Resveratrol supplementation does not improve metabolic function in nonobese women with normal glucose tolerance. Cell Metabolism 16: 658-664.

*Corresponding author: Michael B. Zemel, Ph.D, Professor Emeritus, The University of Tennessee, Chief Scientific Officer, NuSirt Sciences, Inc. 11020 Solway School Road, Suite 109, Knoxville, Tennessee 37923, USA, Tel: +865206-6154; E-mail: mzemel@nusirt.com

Received December 21, 2012; Accepted December 21, 2012; Published December 24, 2012

Citation: Zemel MB (2013) Dietary Supplements in Management and Prevention of Chronic Disease. J Nutr Disorders Ther 3:e107. doi:10.4172/2161-0509.1000e107

Copyright: @ 2013 Zemel MB. This is an open-access article distributed under the terms of the Creative Commons Attribution License, which permits unrestricted use, distribution, and reproduction in any medium, provided the original author and source are credited. 